\title{
Zielfindung leicht gemacht
}

Die Implementierung betrieblicher Umweltschutzinstrumente hat zu einer Erhöhung der Ökoeffizienz der Unternehmen beigetragen. Umweltentlastungen sind jedoch in stärkerem Ausmaß von der Ökoeffektivität, also der ökologischen Wirksamkeit, abhängig. Diese wurde bislang kaum in Betracht gezogen. In einem IÖW-Forschungsvorhaben wurde eine Methode entwickelt, die dieses Defizit beheben soll.

S Von Kathrin Ankele eit Ende der achtziger Jahre findet eine kontinuierliche Entwicklung von Methoden und Instrumenten des betrieblichen Umweltschutzes statt. Die Herangehensweise wird dabei immer systematischer. Die EG-Öko-Audit-Verordnung oder die Internationale Umweltmanagement-Norm ISO 14001 sind nur zwei Beispiele hierfür. Evaluationsvorhaben über die ökologischen Wirkungen des betrieblichen Umweltmanagements haben gezeigt, dass dabei überwiegend nach dem Maßstab der Ökoeffizienz vorgegangen wird (1). Die Ökoeffektivität spielt dagegen eine untergeordnete Rolle. Dies liegt unter anderem daran, dass Unternehmen in der Regel den Unterschied zwischen Emissionen und Umweltauswirkungen nicht kennen und sie sich somit nicht damit auseinandersetzen. Vor diesem Hintergrund wurde 1999 in einem IöWForschungsprojekt im Rahmen des Forschungsprogramms BWPLUS des Landes Baden-Württemberg eine Methode entwickelt, die den Maßstab der Ökoeffektivität in das betriebliche Kalkül einführt. Diese Methode soll hier kurz beschrieben werden (2).

Die Beschäftigung mit Ökoeffektivität und Umweltauswirkungen liegt außerhalb der üblichen unternehmerischen Tätigkeiten. Weshalb sollten Unternehmen es dennoch tun? Einerseits aus Gründen rationaler Zweckbindung: die betrieblichen Mittel für Umweltschutz sollten dort eingesetzt werden, wo sie die größten positiven Wirkungen erzielen. Und diese sollten nicht nur im Bereich der Kostensenkungspotenziale liegen - so legitim und im Rahmen der Systemlogik eines Unternehmens rational diese Zielsetzung auch ist. Die tatsächlichen Umweltentlastungen sollten dabei nicht aus dem Blick geraten. So trivial es klingt: Die Unternehmen als gesellschaftliches Subsystem tragen eben auch Verantwortung für die Erhaltung der natürlichen
Lebensgrundlagen (neben anderen Akteuren). Eine gesetzliche Verpflichtung zu einer solchen Herangehensweise besteht jedoch nicht. Die Methode ist daher für diejenigen Unternehmen gedacht, die sich dieser Verantwortung bewusst sind und mit der rein stoffflussorientierten Herangehensweise an Grenzen gestoßen sind.

\section{Ausgangspunkt Stoffflüsse}

Ausgangspunkt der Methode ist die Überprüfung der Frage, ob aus den betrieblichen Tätigkeiten Emissionen resultieren, die zu bestimmten lokalen, regionalen und globalen Umweltauswirkungen beitragen. Hierfür kann eine im Projekt entwickelte Tabelle herangezogen werden, die eine exemplarische Auswahl an betrieblichen Stoff- und Energieflüssen sowie an Umweltauswirkungen als Folge dieser Stoffflüsse enthält. Beispiele hierfür sind Treibhauseffekt, Ozonabbau, Eutrophierung oder Ökotoxizität. Die betrieblichen Daten sollten möglichst nicht nur aggregiert für das gesamte Unternehmen ermittelt, sondern auch nach Bereichen und Funktionen aufgeschlüsselt werden. Auf diese Weise können die zu entwickelnden Umweltziele und Maßnahmen direkt am Entstehungsort ansetzen. Zugleich kann stichhaltiger abgeschätzt werden, welche Einsparungen erreichbar sind.

\section{Umweltauswirkungen gewichten}

Daraufhin wird gepruift, ob die jeweilige Umweltauswirkung im lokalen oder regionalen Bezugsraum des Betriebsstandortes von Bedeutung ist. Für viele Umweltauswirkungen liegen Karten vor, in denen farblich hervorgehoben ist, ob und in welchem Maße Grenzwerte überschritten sind. Diese Frage kann somit auf einen Blick beantwortet werden (3).

Umweltauswirkungen gehen auf Emissionen unterschiedlicher Quellen zurïck. Ein Hinweis darauf, wie hoch der Anteil von Industrie und
Gewerbe ist und welcher Handlungsbedarf somit besteht, kann Emissionskatastern entnommen werden. Diese weisen den Anteil von Industrie und Gewerbe im Vergleich zu anderen Quellen wie Kleinfeuerungsanlagen, Verkehr und biogenen Quellen aus.

Die Unterteilung ist grob und hochaggregiert, könnte bei Bedarf jedoch weiter differenziert werden, da bei den statistischen Landesämtern die entsprechenden Daten vorliegen. Das Emissionskataster von Baden-Württemberg enthält anschauliche Tortendiagramme für das gesamte Bundesland und differenziert nach Land- und Stadtkreisen, die den Anteil der genannten Sektoren an den Umweltauswirkungen beziehungsweise den zugrunde liegenden Emissionen ausweisen. Hier muss auf Emissionen zurïckgegriffen werden, da bislang keine Aggregation zu Umweltauswirkungen erfolgt.

Die Betrachtung der Belastungssituation im Bezugsraum des Standortes darf selbst bei der Unterschreitung von Grenzwerten jedoch nicht signalisieren, dass eine „Auffïllstrategie“ bis zum Grenzwert verfolgt werden kann. Um dies zu verhindern, wird geprüft welche branchenbedingten und/oder unternehmensspezifischen Emissionsschwerpunkte bestehen. Dazu können zum Beispiel Branchenleitfäden herangezogen werden. Auch Benchmarking-Ansätze können hierfür sehr hilfreich sein. Dies ermöglicht jedem Unternehmen eine Selbsteinschätzung des individuellen Risikos, branchenüblichen Umweltschutzstandards nicht zu genügen und mittelfristig möglicherweise mit behördlichen Auflagen oder öffentlichem Druck konfrontiert zu werden. Auf diese Weise werden unabhängig von der Immissionssituation Ansatzpunkte für betriebliche 0ptimierungen im Sinne der Risikovorsorge ermittelt.

\section{Bezug zu Umweltzielen}

Richtung und Dringlichkeit von Umweltschutzmaßnahmen können anhand der Entwicklung des Umweltzustandes im Vergleich zu Umweltqualitäts- und -handlungszielen abgeschätzt werden. Die Entwicklung des Umweltzustandes wird mit Umweltindikatoren dargestellt. Beispielsweise wurde in den Umweltdaten 95/96 von BadenWürttemberg eine Zusammenstellung von 16 Umweltindikatoren veröffentlicht, die einen Überblick über die Umweltsituation seit Ende der 70er Jahre vermitteln sollen (4). Nimmt das Ausmaß der Umweltauswirkung zu, kann in der Regel davon ausgegangen werden, dass Handlungsbedarf geboten ist. 
Die Interpretation des Trends sollte durch den Einbezug von im politischen Prozess festgelegten Umwelthandlungszielen erfolgen, die als Orientierung für das Ausmaß der erforderlichen Reduktion betrachtet werden können. Mit diesen Hintergrundinformationen kann das einzelne Unternehmen vergleichend abschätzen, welche Reduktionen generell erforderlich sind und welchen individuellen Beitrag es dazu leisten kann und will

\section{Bildung eines Gesamturteils}

Die qualitativen Einzelbewertungen der vorangegangenen Schritte werden nun zu einem Gesamturteil für jede Umweltauswirkung zusammengefasst, um den betrieblichen Handlungsbedarf zu ermitteln. Die Relevanz des Sektors Industrie, Emissionsschwerpunkte der Branche mit Bezug zu der jeweiligen Umweltauswirkung und die Relevanz der Umweltauswirkung im Bezugsraum werden auf einer Stufenskala mit hoch, mittel, gering oder mit Zwischenstufen beurteilt. Diese Informationen werden dann unter Einbezug der Richtung des Trends (steigend, stagnierend, sinkend) und der Höhe definierter Umwelthandlungsziele, die als Orientierung für die angestrebte Gesamtreduktion dient, zu einem Gesamturteil Handlungsbedarf mit den Prioritäten hoch, mittel oder gering zusammengefasst.

Hierfür müssen die Einzelaspekte untereinander gewichtet werden. Die Gewichtung bleibt den Anwendern überlassen, da sich darin die Umweltpolitik des Unternehmens niederschlägt. Je nachdem, ob diese Politik einen eher proaktiven Ansatz zur Umweltentlastung oder aber eine eher defensive Strategie der Anpassung an den unteren Durchschnitt verfolgt, werden die Gewichtungen unterschiedlich ausfallen. Insofern ist eine Standardisierung dieses Schrittes nicht sinnvoll, auch wenn es anfänglich ungewohnt und schwierig erscheinen mag.

Abbildung 1 fasst die einzelnen Schritte der Methode zusammen.

\section{- Weiterer Handlungsbedarf}

Die Einbeziehung der Umweltsituation und bestehender Umwelthandlungsziele im Rahmen des betrieblichen Umweltschutzes stellt keine wissenschaftliche Fingerübung dar. Vielmehr geht es um den Nachweis der Wirksamkeit und Sinnhaftigkeit betrieblicher Umweltschutzmaßnahmen durch Erzielung von Umweltentlastungen. Die Entwicklung betrieblicher Umwelt-

\section{Abbildung 1: Schritte der regelmäBigen Bewertung}

Zuordnung betrieblicher Stoffflüsse zu lokalen, regionalen und globalen Umweltauswirkungen

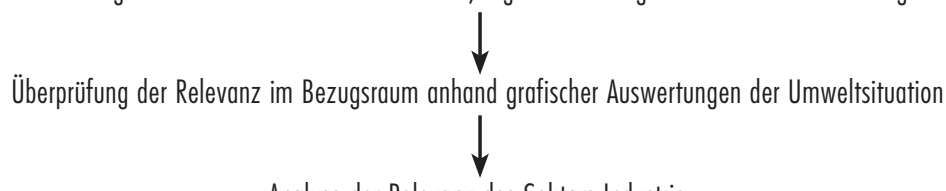

Analyse der Relevanz des Sektors Industrie

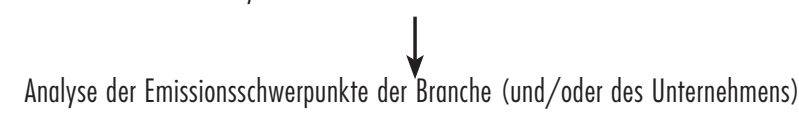

Abschätzung des Handlungsbedarfs anhand des Trends ausgewählter Umweltindikatoren

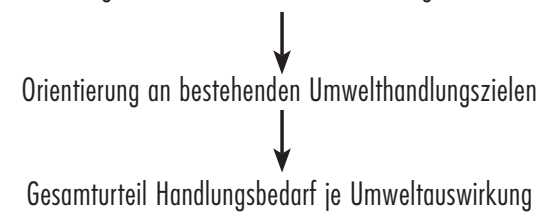

Festlegen von Prioritäten und Umweltschutzzielen, Rückschlüsse auf betriebliche Prozesse

$$
\text { und Tätigkeiten zur Maßnahmenformulierung }
$$

Quelle: eigene Darstellung

schutzziele und -maßnahmen auf der Basis der beschriebenen Vorgehensweise kann als Baustein einer Umweltpolitik verstanden werden, in der der Staat über Rahmenbedingungen die Ziele steuert und Unternehmen im Rahmen ihrer betriebswirtschaftlichen Systemlogik (Mit-)Verantwortung für die Zielerreichung übernehmen. Das entspricht auch den seit einigen Jahren von beiden Seiten formulierten Erwartungen. Mit Blick auf die Methodenanwendung besteht weiterer Entwicklungsbedarf hinsichtlich der Verfügbarkeit und Anschaulichkeit der erforderlichen Umweltdaten, Umweltindikatoren und Umwelthandlungsziele. Im Projekt wurden Vorschläge zur Weiterentwicklung, Darstellung und Bereitstellung dieser Daten durch die Behörden gemacht. Die Weiterleitung an die Unternehmen sollte durch Kammern und Verbände erfolgen. Darüber hinaus benötigen insbesondere kleine und mittlere Unternehmen weitgehende Unterstiitzung in Bezug auf das Methoden- und Bewertungs-Know-how. Auch hierfür wurde im Projekt ein Vorschlag erarbeitet, der Kammern und Verbände einbezieht.

\section{Anmerkungen}

(1) Vgl. Umweltbundesamt (Hrsg.): Umweltmanagement in der Praxis, Teilergebnisse zur Vorbereitung der 1998 vorgesehenen Überprüfung des gemeinschaftlichen Öko-Audit-Systems. UBA-Texte 20/98 (Teile I bis III) und 52/98 (Teile IV bis VI), Berlin 1998, und
Ankele, K. et al.: Fachwissenschaftliche Untersuchung der Wirksamkeit der EG-Öko-Audit-Verordnung. In: UmweltWirtschaftsForum, Nr. 4 / 1998.

(2) Die Methode wird ausführlich beschrieben in Ankele, K./ Kottmann, H. (unter Mitarbeit von A.Volkens): Ökologische Zielfindung im Rahmen des Umweltmanagements. Entwicklung einer Methode für die Unternehmenspraxis. IÖW-Schriftenreihe 147/00, Berlin (im Erscheinen).

(3) Vergleiche hierzu auch den Leiffaden Umweltbundesamt (Hrsg.): Betriebliche Umweltauswirkungen - ihre

Erfassung und Bewertung im Rahmen des Umweltmanagements, Berlin 1999.

(4) Landesanstalt für Umweltschutz Baden-Württemberg/ Umwelt- und Verkehrsministerium Baden-Württemberg: Umweltdaten 95/96. Karlsruhe 1997 (erscheint regelmäBig).

\section{Die Autorin}

Kathrin Ankele ist wissenschaftliche Mitarbeiterin im Forschungsfeld Ökologische Unternehmenspolitik des IÖW.

Kontakt: IÖW, Potsdamer Str. 105, 10785 Berlin. Tel. 030/884594-0, Fax 030/8825439, E-mail: Kathrin.Ankele@ioew.de 
(c) 20I0 Authors; licensee IÖW and oekom verlag. This is an article distributed under the terms of the Creative Commons Attribution Non-Commercial No Derivates License (http://creativecommons.org/licenses/by-nc-nd/3.o/), which permits unrestricted use, distribution, and reproduction in any medium, provided the original work is properly cited. 